\title{
Small-Scale Crisis in Cosmology - Sterile Neutrinos to the Rescue?
}

\author{
Jörn Kersten* \\ University of Bergen, Norway \\ E-mail: joern.kersten@uib.no
}

The $\Lambda$ CDM standard model of cosmology is in excellent agreement with data on large scales but has difficulty explaining all observations on small scales. I discuss a simple particle physics model involving a new MeV-scale gauge boson that mediates dark matter self-interactions and dark matter interactions with a new light fermion. This allows to solve all small-scale problems of $\Lambda C D M$ cosmology. It would be very attractive to identify the new light particle with a sterile neutrino, but it appears that this option is disfavored.

Neutrino Oscillation Workshop (NOW2018)

9-16 September, 2018

Rosa Marina (Ostuni, Brindisi, Italy)

\footnotetext{
${ }^{*}$ Speaker.
} 


\section{Introduction}

Ever more precise observations probe the $\Lambda \mathrm{CDM}$ standard model of cosmology, a flat universe dominated by a cosmological constant and non-baryonic cold dark matter (DM), and seemingly confirm this relatively minimal scenario. For example, the power spectrum of the cosmic microwave background is in excellent agreement with $\Lambda \mathrm{CDM}$ [1], and the distribution of galaxies predicted by numerical simulations of structure formation closely matches actual observations [2]. However, looking more closely one realizes that these observations probe cosmology at large scales. On small scales, i.e., in observations of dwarf galaxies, the scenario faces significant challenges [3]. The number of small satellite galaxies predicted for a Milky-Way-size galaxy in $\Lambda \mathrm{CDM}$ far exceeds the number of known Milky Way satellites (missing satellite problem). Small, DM-dominated galaxies seem to possess cored inner density profiles, which means that the density becomes nearly constant towards the center. This is inconsistent with the $\Lambda \mathrm{CDM}$ prediction of cuspy profiles, where the density is inversely proportional to the distance from the center (cusp-core problem). Comparing the brightest observed satellites of the Milky Way with the most massive satellites found in $\Lambda \mathrm{CDM}$ simulations, which should be too big to fail at forming stars, one finds that the central regions of the simulated dwarf galaxies are more concentrated than their observed counterparts (too-big-to-fail problem). An important caveat is that the simulations used to identify these problems considered only cold DM. It is therefore possible that the simulations can be brought into agreement with observations by including the matter made of Standard Model baryons. However, this possibility is called into question by the discovery that refined simulations which do include baryons lead to an agreement between simulated and observed dwarf rotation curves in some cases while discrepancies remain in others (diversity problem) [4]. That said, it certainly remains possible, and probably even likely, that the small-scale problems of $\Lambda \mathrm{CDM}$ will eventually be explained by effects from the domain of astrophysics. Nevertheless, it is interesting to wonder if and how they can be addressed by new particle physics, which is the focus of this contribution.

\section{Dark Matter Interacting with Itself and with Dark Radiation}

The approach presented here employs a combination of DM self-interactions and late kinetic decoupling due to interactions between DM and dark radiation. Viable simplified models were classified in [5] but we will focus on a particular model first proposed in [6]. It includes a dark sector consisting of an $\mathrm{MeV}$-scale gauge boson $V$ (dark photon), a scalar $\Theta$, a vector-like DM fermion $\chi$, and chiral fermions $N$ and $N^{\prime}$ with eV-scale and $\mathrm{MeV}$-scale masses, respectively. The boson mediates a $U(1)_{X}$ gauge interaction, which is spontaneously broken by the vacuum expectation value $\langle\Theta\rangle \sim \mathrm{MeV}$. The dark fermions are charged under $U(1)_{X}$ and uncharged under the Standard Model gauge group, while the Standard Model particles are neutral under $U(1)_{X}$. In order to cancel anomalies, we assign charges $X_{N}$ and $X_{N^{\prime}}=-X_{N}$ to the chiral dark fermions.

Effective operators that can be generated by the exchange of heavy singlet fermions lead to Majorana masses for the chiral dark fermions and to their mixing with the Standard Model neutrinos after $U(1)_{X}$ is broken. ${ }^{1}$ We choose parameters such that $m_{N} \sim \mathrm{eV}$ and $m_{N^{\prime}} \sim \mathrm{MeV}$.

\footnotetext{
${ }^{1}$ The original model [6] includes a second dark scalar, but this is not necessary [7].
} 
For our purposes, the terms of interest in the Lagrangian are

$$
\mathscr{L} \supset-g_{X} V_{\mu}\left(\bar{\chi} \gamma^{\mu} \chi+X_{N} \bar{N} \gamma^{\mu} N-X_{N} \overline{N^{\prime}} \gamma^{\mu} N^{\prime}\right)+\kappa|H|^{2}|\Theta|^{2},
$$

where $g_{X}$ denotes the $U(1)_{X}$ gauge coupling. Apart from the gauge couplings of the dark fermions, there is a portal coupling between the dark scalar $\Theta$ and the Standard Model Higgs $H$. This coupling ensures that the dark sector is in thermal equilibrium with the Standard Model particles in the very early universe. The DM relic density is thus obtained by the standard freeze-out mechanism. Setting it equal to the observed value determines $g_{X}$ as a function of the DM mass $m_{\mathrm{DM}}$.

If the dark gauge boson is much lighter than the DM, it mediates a long-range DM selfinteraction. Its strength depends on the velocity of the DM particles. Hence, on the one hand we can obtain sufficiently weak self-interactions for the large velocities occurring on the scale of galaxy clusters, where stringent bounds exist. On the other hand, in dwarf galaxies, where much smaller velocities are typical, the self-interaction cross section can become large, $\sigma / m_{\mathrm{DM}} \sim 1 \mathrm{~cm}^{2} / \mathrm{g}$. This enables a solution of the cusp-core, too-big-to-fail, and diversity problems $[8,9,10,11,12]$.

The light chiral fermion $N$ stays relativistic in the early universe until the temperature falls below its mass. Thus, it acts as dark radiation (a relativistic degree of freedom different from photons and Standard Model neutrinos) for $T \gtrsim 1 \mathrm{eV}$. The new gauge interaction leads to efficient scattering between the DM and the dark radiation, which causes the two species to stay in kinetic equilibrium long after the chemical decoupling (freeze-out) of the DM around $T \sim m_{\mathrm{DM}} / 25$. As a consequence, structure formation is suppressed at small scales [13, 14, 15]. This solves the missing satellites problem if the kinetic decoupling between DM and dark radiation occurs at a temperature $T_{\mathrm{kd}}$ slightly below $1 \mathrm{keV}[13,16]$. One should caution that the suppression of smallscale structure is constrained by observations of the Lyman- $\alpha$ forest, analogous to the warm DM scenario. A recent study concluded that this allows a reduction of the number of Milky Way satellites by at most $30 \%$ [17]. If this result stands, the model presented here can still contribute to solving the missing satellites problem, but additional contributions from astrophysics are required.

With this qualitative understanding of the features of the model, we can now discuss which regions in parameter space provide solutions to the small-scale problems of $\Lambda \mathrm{CDM}$. As the dark gauge coupling is fixed by the observed DM density and as the mass of the dark radiation particle is only weakly constrained by the requirement $m_{N} \ll T_{\mathrm{kd}}$, the relevant free parameters are the DM mass, the mass $m_{\text {med }}$ of the gauge boson mediating the $U(1)_{X}$ interaction, and the $U(1)_{X}$ charge $X_{N}$. For $X_{N}=1$, the parameter space region in which the missing satellites problem is solved is shown by the green bands in figure 1 . Smaller values of $X_{N}$ weaken the interaction between DM and dark radiation, which has to be compensated by lowering the dark photon mass if the impact on structure formation is to stay the same; consequently, the green bands move to the left. The blue bands in the figure indicate the parameter space region where DM self-interactions have the right strength to solve the cusp-core, too-big-to-fail, and diversity problems. As the different bands overlap, all small-scale problems can be addressed simultaneously. For $X_{N}=1$, this happens if the dark photon mass is of order $\mathrm{MeV}$ and if the order of magnitude of the DM mass is either a $\mathrm{TeV}$ or a few hundred MeV. The region with lighter DM may be favored by observations of galaxy clusters [17].

The light chiral fermions $N$ increase the energy density of relativistic particles in the early universe, which is parameterized by the effective number of neutrino species $N_{\text {eff. }}$. Assuming that no other dark-sector particles remain relativistic during big bang nucleosynthesis, we find $\Delta N_{\text {eff }} \gtrsim$ 


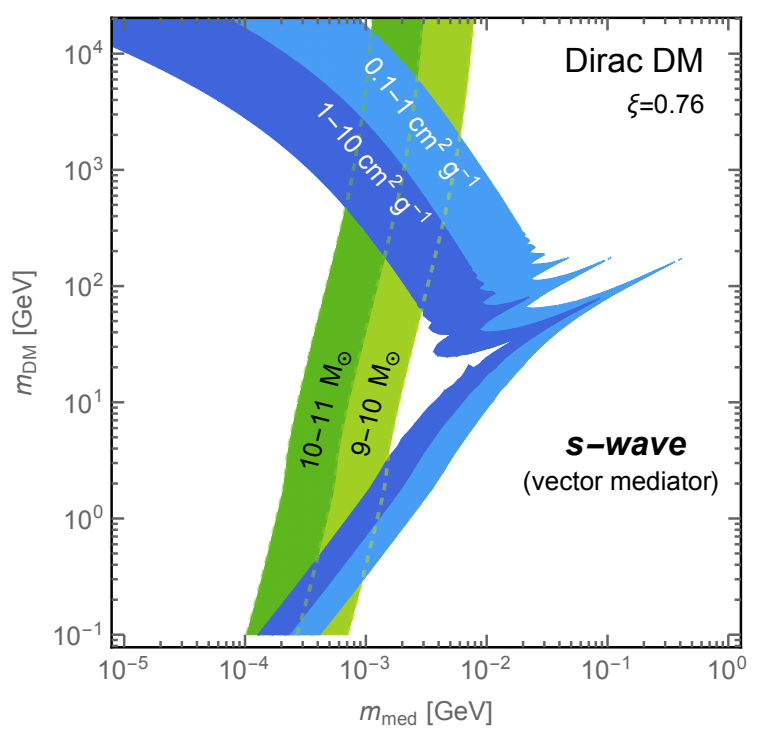

Figure 1: Parameter space regions yielding the correct DM relic density as well as a solution of the missing satellites (green bands) and other small-scale problems (blue bands), as determined by DarkSUSY 6.1 [18]. The numbers in the green band indicate the mass cutoff below which no galaxies can form (and $9 M_{\odot}$ means $10^{9} M_{\odot}$, for example). The numbers in the blue bands correspond to the DM self-interaction cross section at dwarf-galaxy scales divided by the DM mass.

0.33. On the one hand, this is of the correct order of magnitude to address tensions between measurements of the Hubble parameter $H_{0}$ and the magnitude of matter density fluctuations $\sigma_{8}$ at different redshifts [19]. On the other hand, it is in tension with big bang nucleosynthesis constraints at the $2 \sigma$ level [20].

\section{Sterile Neutrinos as Dark Radiation}

A tempting possibility is identifying the new eV-scale fermion with the sterile neutrino associated with the anomalies observed in experiments such as LSND and MiniBooNE [21, 22]. A standard sterile neutrino with $\mathscr{O}(1 \mathrm{eV})$ mass and $\mathscr{O}(0.1)$ mixing with the Standard Model neutrinos, as indicated by the anomalies, is strongly disfavored by cosmology since it is copiously produced by oscillations in the early universe, which violates the bound on the sum of neutrino masses, $\sum m_{v} \lesssim 0.1 \mathrm{eV}$ [1]. However, in our scenario the $U(1)_{X}$ interaction of the sterile neutrinos $N$ leads to an effective matter potential that suppresses active-sterile neutrino mixing and thus sterile neutrino production at temperatures above an $\mathrm{MeV}[23,24]$. Hence, there is a chance to avoid the cosmological neutrino mass bound.

At temperatures below the mass of the dark photon, however, the $U(1)_{X}$ interactions become weaker, and consequently the active-sterile neutrino mixing returns to its unsuppressed vacuum value. Then additional sterile neutrino production occurs [6], which is sufficient to equalize the temperatures of Standard Model and sterile neutrinos [25, 26]. An interesting detail of this rethermalization or recoupling process is that it does not conserve entropy, contrary to claims in some works, including [6]. One way to see this is realizing that the process is irreversible, which implies 
an increase in entropy. Instead, the total neutrino number and energy densities are conserved. As a consequence, the momentum distribution functions are characterized by a common kinetic temperature and a non-vanishing chemical potential [27].

A number of studies considered re-thermalization and its impact on cosmology, arriving at varying conclusions [25, 26, 27, 28, 29, 30]. The most recent work [30] found that a sterile neutrino with significant mixing with the Standard Model neutrinos is not a viable candidate for the dark radiation particle. Depending on the values of the model parameters, the reason is either that the bound on the sum of neutrino masses is violated or that neutrino free-streaming is too much reduced to be compatible with cosmic microwave background observations.

\section{Conclusions}

Discrepancies between observations of dwarf galaxies and simulations of structure formation may point to deviations from the standard picture of collisionless cold dark matter. I have presented a model that introduces a new gauge interaction mediated by an MeV-scale gauge boson. This leads to dark matter self-interactions solving the cusp-core, too-big-to-fail, and diversity problems. In addition, a new eV-scale particle species interacts with the dark matter by the new force, which results in the suppression of structure formation at small scales, solving the missing satellites problem. Thus, a simple particle physics model is able to address all small-scale problems of $\Lambda \mathrm{CDM}$ cosmology.

It would be tempting to identify the new eV-scale fermion with a sterile neutrino that mixes with the Standard Model neutrinos and explains the anomalies observed in oscillation experiments. However, this option does not seem to be compatible with constraints from cosmology. In this case, the new light particles have to be generic dark fermions that mix at most very weakly with Standard Model neutrinos.

\section{Acknowledgements}

I would like to thank Torsten Bringmann for the collaboration on self-interacting dark matter model building and for providing the plot in figure 1. I am grateful to Alessandro Mirizzi, Basudeb Dasgupta, Joachim Kopp, and Rasmus Hansen for very helpful discussions during the workshop. Besides, I thank the Abdus Salam ICTP for hospitality during the writing of these proceedings and acknowledge travel support from the Research Council of Norway under project number 255182. Special thanks are due to the NOW organizers, in particular Eligio Lisi, for their truly outstanding organization and hospitality.

\section{References}

[1] Planck collaboration, N. Aghanim et al., Planck 2018 results. VI. Cosmological parameters, 1807.06209.

[2] V. Springel, C. S. Frenk and S. D. M. White, The large-scale structure of the Universe, Nature 440 (2006) 1137 [astro-ph/0604561].

[3] J. S. Bullock and M. Boylan-Kolchin, Small-Scale Challenges to the $\Lambda$ CDM Paradigm, Ann. Rev. Astron. Astrophys. 55 (2017) 343 [1707.04256]. 
[4] K. A. Oman et al., The unexpected diversity of dwarf galaxy rotation curves, Mon. Not. Roy. Astron. Soc. 452 (2015) 3650 [1504.01437].

[5] T. Bringmann, H. T. Ihle, J. Kersten and P. Walia, Suppressing structure formation at dwarf galaxy scales and below: late kinetic decoupling as a compelling alternative to warm dark matter, Phys. Rev. D94 (2016) 103529 [1603.04884].

[6] T. Bringmann, J. Hasenkamp and J. Kersten, Tight bonds between sterile neutrinos and dark matter, JCAP 1407 (2014) 042 [1312.4947].

[7] J. F. Cherry, A. Friedland and I. M. Shoemaker, Neutrino Portal Dark Matter: From Dwarf Galaxies to IceCube, 1411.1071.

[8] J. L. Feng, M. Kaplinghat and H.-B. Yu, Halo Shape and Relic Density Exclusions of Sommerfeld-Enhanced Dark Matter Explanations of Cosmic Ray Excesses, Phys. Rev. Lett. 104 (2010) 151301 [0911.0422].

[9] A. Loeb and N. Weiner, Cores in Dwarf Galaxies from Dark Matter with a Yukawa Potential, Phys. Rev. Lett. 106 (2011) 171302 [1011.6374].

[10] M. Vogelsberger, J. Zavala and A. Loeb, Subhaloes in Self-Interacting Galactic Dark Matter Haloes, Mon. Not. Roy. Astron. Soc. 423 (2012) 3740 [1201 . 5892].

[11] M. Valli and H.-B. Yu, Dark matter self-interactions from the internal dynamics of dwarf spheroidals, Nat. Astron. 2 (2018) 907 [1711.03502].

[12] A. Kamada, M. Kaplinghat, A. B. Pace and H.-B. Yu, How the Self-Interacting Dark Matter Model Explains the Diverse Galactic Rotation Curves, Phys. Rev. Lett. 119 (2017) 111102 [1611.02716].

[13] C. Boehm, P. Fayet and R. Schaeffer, Constraining dark matter candidates from structure formation, Phys. Lett. B518 (2001) 8 [astro-ph/0012504].

[14] A. M. Green, S. Hofmann and D. J. Schwarz, The first WIMPy halos, JCAP 08 (2005) 003 [astro-ph/0503387].

[15] A. Loeb and M. Zaldarriaga, The Small-scale power spectrum of cold dark matter, Phys. Rev. D71 (2005) 103520 [astro-ph/0 504112 ].

[16] L. G. van den Aarssen, T. Bringmann and C. Pfrommer, Is dark matter with long-range interactions a solution to all small-scale problems of $\Lambda$ CDM cosmology?, Phys. Rev. Lett. 109 (2012) 231301 [1205.5809].

[17] R. Huo, M. Kaplinghat, Z. Pan and H.-B. Yu, Signatures of self-interacting dark matter in the matter power spectrum and the CMB, Phys. Lett. B783 (2018) 76 [1709. 09717].

[18] T. Bringmann, J. Edsjö, P. Gondolo, P. Ullio and L. Bergström, DarkSUSY 6: an advanced tool to compute dark matter properties numerically, JCAP 07 (2018) 033 [1802.03399].

[19] S. Roy Choudhury and S. Choubey, Constraining light sterile neutrino mass with the BICEP2/Keck Array 2014 B-mode polarization data, 1807.10294.

[20] M. Hufnagel, K. Schmidt-Hoberg and S. Wild, BBN constraints on MeV-scale dark sectors. Part I. Sterile decays, JCAP 02 (2018) 044 [1712.03972].

[21] LSND collaboration, A. Aguilar-Arevalo et al., Evidence for neutrino oscillations from the observation of $\bar{v}_{e}$ appearance in a $\bar{v}_{\mu}$ beam, Phys. Rev. D64 (2001) 112007 [hep-ex/ 0104049 ]. 
[22] MiniBoonE collaboration, A. A. Aguilar-Arevalo et al., Significant Excess of Electronlike Events in the MiniBooNE Short-Baseline Neutrino Experiment, Phys. Rev. Lett. 121 (2018) 221801 [1805.12028].

[23] S. Hannestad, R. S. Hansen and T. Tram, How Self-Interactions can Reconcile Sterile Neutrinos with Cosmology, Phys. Rev. Lett. 112 (2014) 031802 [1310 . 5926].

[24] B. Dasgupta and J. Kopp, Cosmologically Safe eV-Scale Sterile Neutrinos and Improved Dark Matter Structure, Phys. Rev. Lett. 112 (2014) 031803 [1310.6337].

[25] A. Mirizzi, G. Mangano, O. Pisanti and N. Saviano, Collisional production of sterile neutrinos via secret interactions and cosmological implications, Phys. Rev. D91 (2015) 025019 [1410.1385].

[26] Y. Tang, More Is Different: Reconciling eV Sterile Neutrinos with Cosmological Mass Bounds, Phys. Lett. B750 (2015) 201 [1501.00059].

[27] X. Chu, B. Dasgupta and J. Kopp, Sterile neutrinos with secret interactions - lasting friendship with cosmology, JCAP 10 (2015) 011 [1505.02795].

[28] J. F. Cherry, A. Friedland and I. M. Shoemaker, Short-baseline neutrino oscillations, Planck, and IceCube, 1605.06506.

[29] F. Forastieri, M. Lattanzi, G. Mangano, A. Mirizzi, P. Natoli and N. Saviano, Cosmic microwave background constraints on secret interactions among sterile neutrinos, JCAP 07 (2017) 038 [1704.00626].

[30] X. Chu, B. Dasgupta, M. Dentler, J. Kopp and N. Saviano, Sterile Neutrinos with Secret Interactions Cosmological Discord?, JCAP 11 (2018) 049 [1806.10629]. 